\title{
ESTIMATION OF TROPOSPHERIC DELAYS USING SYNTHETIC APERTURE RADAR AND SQUINT DIVERSITY
}

\author{
Marc Rodriguez-Cassola, Pau Prats-Iraola, Marc Jäger, Andreas Reigber, and Alberto Moreira \\ Microwaves and Radar Institute, German Aerospace Center (DLR)
}

\section{ABSTRACT}

This paper proposes a method for extracting the tropospheric time delay in a single-pass using SAR images acquired in squinted geometries. The required accuracies and error sources are analysed and a satellite configuration providing single-pass measurements of the tropospheric delay is proposed. Moreover, a joint topography-tropospheric delay estimation using very-high resolution SAR images is also suggested for cases where an inaccurate DEM is available. A performance of the approach with current TerraSAR-X staring spotlight like parameters is also computed.

\section{INTRODUCTION}

The measurement of the atmosphere plays an important role in the understanding of weather dynamics. Over the years, the SAR community has been particularly interested in measuring the atmospheric behaviour with SAR; examples can be found in the literature, e.g., well-established techniques like repeat-pass interferometry using stacks [1] or recent developments including pursuit monostatic [2], or geostationary systems [3]. We suggest a system capable of delivering the spatial variations of the tropospheric delays operating in single-pass by exploiting squint diversity.

\section{CHARACTERISATION OF THE TROPOSPHERIC ERRORS}

Due to the water vapor content of the atmosphere, the troposphere induces an additional delay on the propagated radar signals. Assuming a zenith observation geometry, this delay is assumed to be dependent on the height of the tropospheric layer, in the form [4]

$$
\Delta r_{\mathrm{ZTD}}(h ; z, H)=z \cdot \exp \left(-\frac{h}{H}\right)
$$

where $z$ is the zenith path delay in meters, $H$ is the height of the layer, and $h$ is the height of the observed target. If the observation geometry is not zenith, as is typically the case of
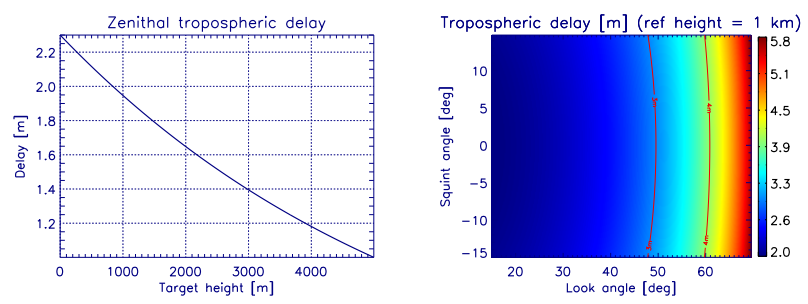

Fig. 1. Example of zenith tropospheric delay as a function of the target height (left). Example of tropospheric delay as a function of the look and squint angles (right). Values used $z=2.3 \mathrm{~m}, H=6000 \mathrm{~m}, h=1000 \mathrm{~m}$.

SAR, the tropospheric delay takes the form [5]

$$
\Delta r_{\mathrm{T}}(\theta, \beta)=\frac{\Delta r_{\mathrm{ZTD}}}{\cos \theta \cdot \cos \beta}=\frac{\Delta r_{\mathrm{BTD}}}{\cos \beta},
$$

where $\theta$ is the look angle of the satellite, $\beta$ is the squint. We denote $\Delta r_{\text {BTD }}$ as the boresight tropospheric delay (BTD). Fig. 1 shows a plot of a typical zenith tropospheric delay scaled to range as a function of the target height (left) and the total tropospheric delay as a function of the look angle and squint angle of the acquisition (right).

\section{MEASURING THE TROPOSPHERIC DELAYS IN SQUINTED OBSERVATIONS}

For small squinted apertures, the evolution of the tropospheric delay along the synthetic aperture has a linear form, which adds to the nominal range history of the target, hence shifting the phase centre of the target response. As a consequence, the focussed response of target is shifted by a certain amount; this shift increases with $\Delta r_{\mathrm{BTD}}$ and with $\beta$; moreover, the magnitude of the azimuth shifts is only slightly dependent on the azimuth bandwidth of the images. Under this assumption, the azimuth shift of the image due to the troposphere can be approximated by

$$
\begin{aligned}
\delta t_{\mathrm{a}, \mathrm{T}} & \approx \frac{r 0}{v^{2}} \cdot \frac{\partial \Delta r_{\mathrm{BTD}}}{\partial t} \approx \frac{\Delta r_{\mathrm{BTD}} \cdot f(\beta) \cdot r_{0}}{v_{\mathrm{e}}^{2}\left(r_{0}, \theta\right)} \\
& =\frac{\Delta r_{\mathrm{BTD}}}{v_{\mathrm{sq}}\left(r_{0}, \beta, \theta\right)}
\end{aligned}
$$


where $r_{0}$ is the range to the target, $v_{\mathrm{e}}$ is the effective velocity, and $f(\beta)$ is a function which increases with $\beta$. As an example, Fig. 2 shows the azimuth shifts introduced by the troposphere in the focussed SAR impulse response as a function of the height of the target and of the squint of the acquisition. The velocity $v_{\mathrm{sq}}$ can be approximated as follows

$$
v_{\mathrm{sq}} \approx \frac{v_{\mathrm{e}}\left(r_{0}, \theta\right)}{2} \cdot \frac{\cos ^{3} \beta}{\sin \beta} ;
$$

which decreases for higher orbits and higher squints. Note that the azimuth shift caused by the troposphere is independent of the carrier wavelength. The estimator of the BTD

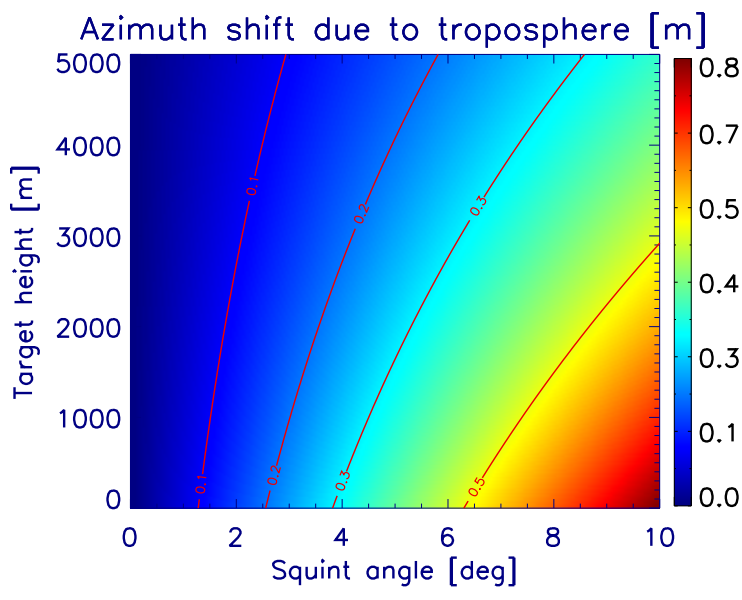

Fig. 2. Azimuth shifts introduced in the SAR impulse response as a function of the target height and the observation squint. Values used $z=2.3 \mathrm{~m}, H=6000 \mathrm{~m}$.

takes the form

$$
\widetilde{\Delta r}_{\mathrm{BTD}}=\left[\widetilde{\frac{\Delta r_{\mathrm{ZTD}}}{\cos \theta}}\right]=\tilde{v}_{\mathrm{sq}} \cdot \widetilde{\delta t_{\mathrm{a}, \mathrm{T}}}
$$

The estimate of $\delta t_{\mathrm{a}, \mathrm{T}}$ is assumed to be unbiased and with variance $\mathrm{E}\left[\widetilde{\delta}_{\mathrm{a}, \mathrm{T}}^{2}\right]$, proportional to the accuracy with which the coregistration offsets can be measured. This accuracy is in general proportional to the azimuth bandwidth of the system, and depends on the way it is measured, e.g., coherent/incoherent cross-correlation or spectral diversity. The term $v_{\mathrm{sq}}$ can be exactly computed if exact geometrical information is available; we however consider that some geometrical errors might arise and therefore use in the estimator $\tilde{v}_{\mathrm{sq}}$ The bias of the estimator in (6) is

$$
\mathrm{E}\left[\widetilde{\Delta r}_{\mathrm{BTD}}-\Delta r_{\mathrm{BTD}}\right] \approx \tilde{v}_{\mathrm{sq}} \cdot \delta t_{\mathrm{res}}+\left(\frac{\tilde{v}_{\mathrm{sq}}}{v_{\mathrm{sq}}}-1\right) \cdot \Delta r_{\mathrm{BTD}},
$$

where $\delta t_{\text {res }}$ represents any residual azimuth shift not due to the tropospheric component. The variance of the estimator in
(6) is

$$
\operatorname{var}\left[\widetilde{\Delta r}_{\mathrm{BTD}}\right] \approx \tilde{v}_{\mathrm{sq}}^{2} \cdot \mathrm{E}\left[\widetilde{\delta t}_{\mathrm{a}, \mathrm{T}}^{2}\right]
$$

The evaluation of (8) presents some operational problems. Due to the nature of the measurement, we cannot rely on coherence between the two images or in the availability of strong point scatterers. The estimation of $\delta t_{\mathrm{a}, \mathrm{T}}$ is clearly a problem of feature tracking, and its accuracy depends both on the scene and the geometry of the observation. We may however accept as valid that

$$
\mathrm{E}\left[\widetilde{\delta}_{\mathrm{a}, \mathrm{T}}^{2}\right] \propto \frac{1}{2 \cdot B_{\mathrm{a}}^{2}}
$$

where $B_{\mathrm{a}}$ is the azimuth bandwidth of the images and the factor 2 is gained from the comparison of the two images. To get an idea of the orders of magnitude we are referring to, let us make some assumptions on the measurement. Since averaging improves the estimation of $\Delta r_{\mathrm{BTD}}$, we will assume an effective averaging of 100, i.e., an improvement factor $k_{\text {int }}=10$ is achievable. Assuming a TerraSAR-X like orbit parameters, an azimuth bandwidth of $8 \mathrm{kHz}$, Fig. 3 shows the accuracy with which the shifts need be measured as a function of the squint of the acquisitions and of the desired accuracy of the BTD. The plotted values scale linearly with $B_{\mathrm{a}}$ and

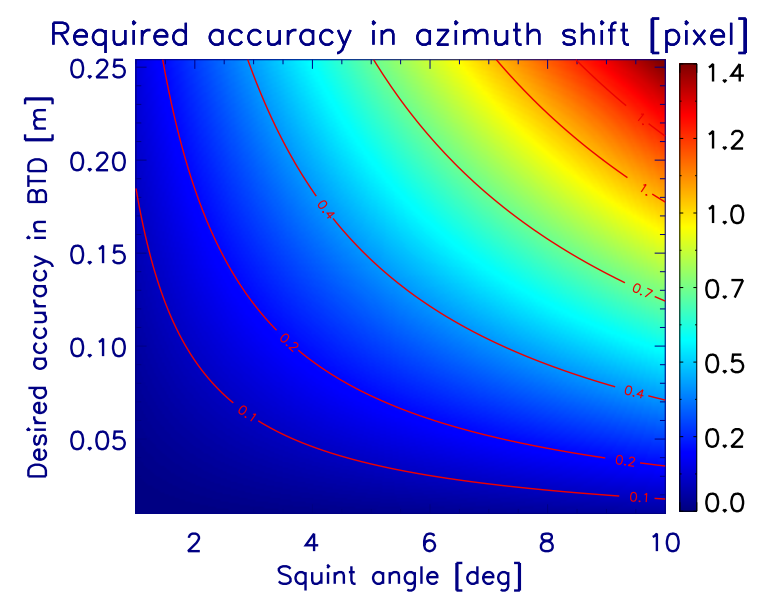

Fig. 3. Required accuracy in the measurement of the azimuth shifts as a function of the squint and of the desired accuracy in the BTD for a single-channel system with $k_{\text {int }}=10$. TerraSAR-X orbit, azimuth bandwidth of $8 \mathrm{kHz}$.

with $k_{\text {int }}$. Higher values of $k_{\text {int }}$ might be achieved if featurerich scenes are observed, or if lower-resolution products are accepted. Due to the lack of expressions describing the performance of feature tracking in SAR images, we cannot link these values to more comprehensible SAR performance parameters. If we assume the feasibility of estimating shifts of about 0.2 pixels, the tropospheric delays could be estimated 
with a $10 \%$ accuracy with squint angles of about $3 \mathrm{deg}$. In the case of TerraSAR-X (squints of up to $2.2 \mathrm{deg}$ ), these values yield accuracies of about $0.25 \mathrm{~m}$. A statistical evaluation with both TerraSAR-X and airborne SAR data remains desirable as future work to understand the behavior of feature tracking algorithms in real scenes for larger squints.

\section{ERROR SOURCES: THE EFFECT OF THE TOPOGRAPHY}

As discussed in the previous section, any additional azimuth shift introduced in the processing stages contaminates the estimate of the tropospheric delay (cf. Eq. (7)). This additional shifts can be due to imperfect calibration and processing errors. Since range calibration accuracies in state-of-theart spaceborne SAR systems are in the order of a few picoseconds [7], we believe processing errors arise as the main limitation for the estimation of tropospheric delays with squint diversity. The habitual cause of residual azimuth shifts introduced in the processing is due to the dependence of the target range history on the height of the target. For a squinted acquisition, the azimuth shift introduced in the processing takes the form [8]

$$
\delta t_{\mathrm{proc}} \approx \frac{f_{\mathrm{DC}} \cdot \lambda \cdot r_{0}}{v_{\mathrm{e}}^{3}} \cdot \Delta v_{\mathrm{e}}(h)
$$

where $f_{\mathrm{DC}}$ is the Doppler centroid and $\Delta v_{\mathrm{e}}$ is the difference between the actual effective velocity of the target and the one used during processing, which is a function of topography. The assumption will be made that the range history of the targets can be locally matched following the accuracy of the available digital elevation model [9]. The variation of the effective velocities with the height change only mildly with the squint, but obviously the Doppler centroid is a direct function of the squint. Given the difficulty to express analytically the variation of the effective velocities with the topography, we prefer a numerical evaluation of the problem. To give an idea of the precision with which the topography must be known, Fig. 4 shows a simulation carried out with a system flying the TerraSAR-X orbit imaging the DLR site at Oberpfaffenhofen with an incident angle of about $35 \mathrm{deg}$. The required precision for the DEM so that the $\delta t_{\text {proc }}$ is kept up to a $10 \%$ of the overall tropospheric delay is shown as a function of the acquisition squint. We note that the values shown in the plot are within the accuracy range of the global TanDEM-X DEM [10].

If topography is known less accurately than required, e.g., in the case of a single-channel system, then a joint estimation topography-troposphere using a high-resolution spotlight acquisition might be suggested. The estimation relies on the detection of coherent scatterers of opportunity within the scene and a perfect calibration of the ranging of the system. By using angular diversity, a linear joint estimation of both parameters can be carried out. Assuming a TerraSAR-X orbit

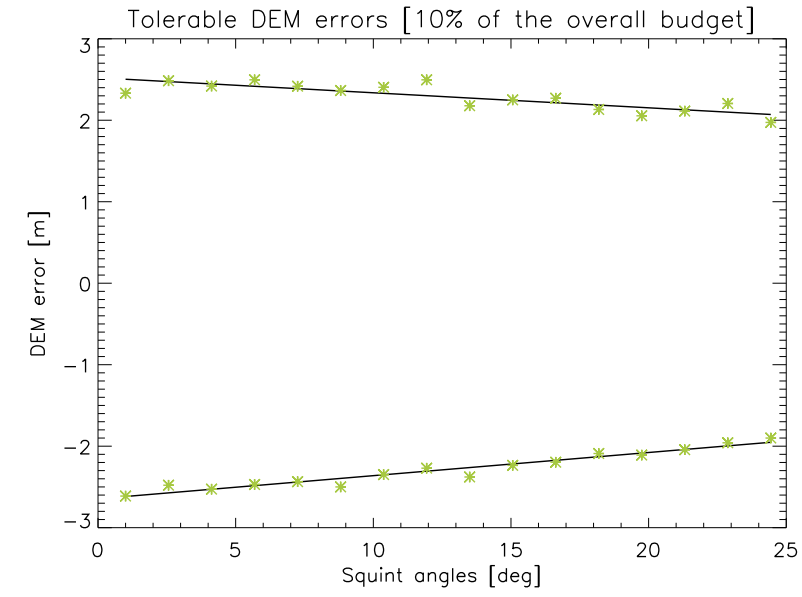

Fig. 4. Required precision in the DEM as a function of the observation squint so that the topography-induced azimuth shifts are kept below the $10 \%$ of the tropospheric shifts. Values used $z=2.3 \mathrm{~m}, H=6000 \mathrm{~m}$.

and $B_{\mathrm{a}}=40 \mathrm{kHz}$, which corresponds to a TerraSAR-X staring spotlight acquisition, the performance of the joint estimate topography-tropospheric delay is shown in Fig. 5. The black lines show the height accuracy, the orange the tropospheric delay accuracy. Solid lines show the performance when the phase error of the CS is available; dotted lines correspond to phase gradient approaches. For the analysed case, and a reference CS with SCR values of about $10 \mathrm{~dB}$, height and tropospheric delay can be estimated with accuracies of $1 \mathrm{~m}$ and $1 \mathrm{~cm}$, respectively. An interesting consequence of the joint estimate topography-troposphere with very-high resolution systems is absolute ranging.

\section{SYSTEM EXAMPLE}

We propose a spaceborne SAR system for measuring the delay of (4) in single-pass operation and a global manner. The approach consists in measuring the azimuth shifts between two consecutive SAR images acquired with different squint angles. Such a system exploiting the squint diversity could be implemented by means of a) a system using a bidirectional antenna pattern [11], and b) a two-system along-track constellation. The geometry of the system is represented in Fig. 6. We remind that the measurement of the tropospheric component from two different squint angles is differential, so a further integration step is required before the tropospheric delay is recovered, in the same manner as in autofocus approaches [12]. The difference between the one-platform and the two-platform system is the time lag between the incremental measurement. Flying on a TerraSAR-X orbit, a system with squints of $\pm 3 \mathrm{deg}$ is expected to provide estimates of the tropospheric delays within $10 \%$ accuracy. Alternatively, 


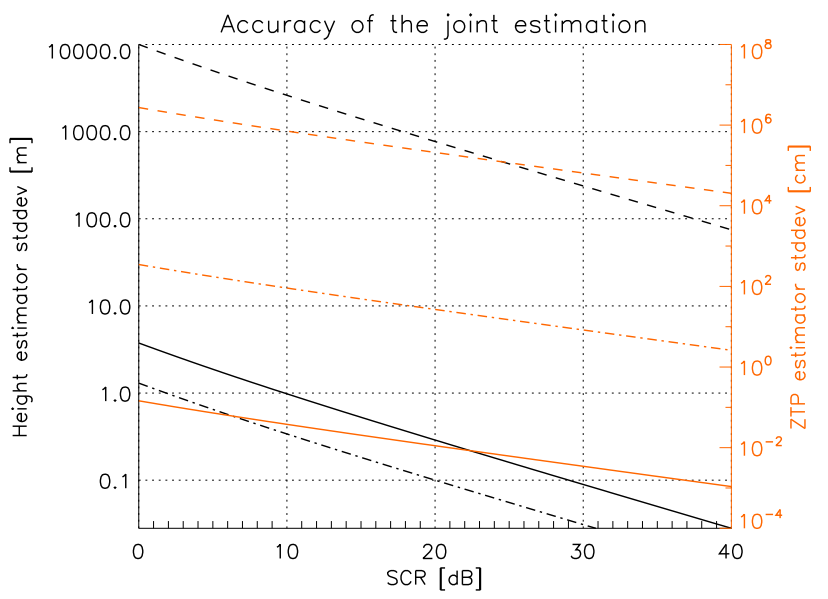

Fig. 5. Accuracy of the joint estimate topographytropospheric delay (zenith tropospheric path, ZTP) based on the exploitation of coherent scatterers for a TerraSAR-X staring spotlight acquisition. The black lines correspond to the height accuracy, the orange to the tropospheric delay accuracy. The solid lines show the performance if a direct measurement of the phase error is available; the dotted lines correspond to phase gradient approaches.

the direct estimation of the phase errors within coherent scatterers could be adopted to avoid the integration step.

\section{SUMMARY}

The paper has presented a method for measuring the atmospheric tropospheric delay in a single-pass using SAR images exploiting squint diversity. With respect to other existing approaches, squint diversity allows us to perform a global measurement of the tropospheric delay in a single pass. An analysis of the accuracy of the method has been presented. For the cases where the estimation of the tropospheric delay is contaminated by a significant DEM error, a joint estimate topography-tropospheric delay with very-high resolution images has been suggested. Present staring spotlight TerraSAR$\mathrm{X}$ acquisitions might already provide very good estimates of both parameters.

\section{REFERENCES}

[1] R. Hanssen, Radar Interferometry: Data Interpretation and Error Analysis, Springer, 2001.

[2] S. Tebaldini et al, 'Recovering time and space varying phase screens through SAR multi-squint differential interferometry,' Proc. EUSAR, 2012.

[3] J. Ruiz-Rodón et al, 'Results on spatial-temporal atmo-

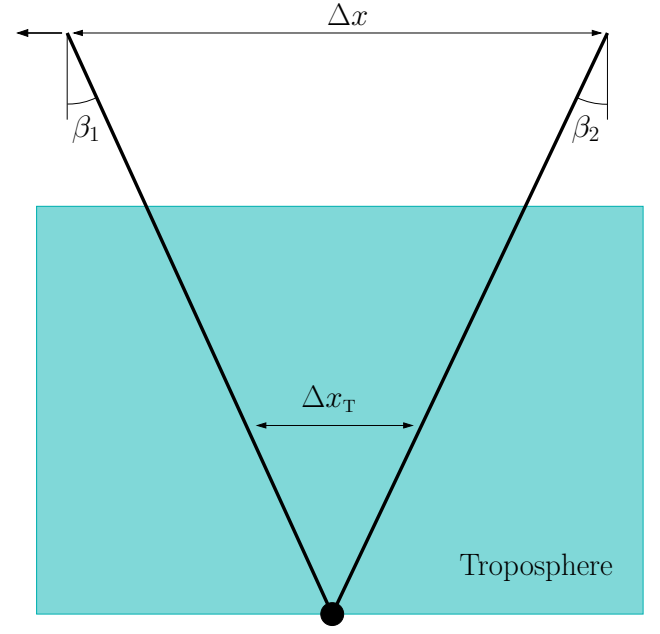

Fig. 6. Example of the geometry used for measuring the topography using squint diversity.

spheric phase screen retrieval from long-term GeoSAR acquisitions,' Proc. IGARSS, 2012.

[4] H. Breit et al, 'TerraSAR-X SAR Processing and Products,' IEEE Trans. Geosci. Remote Sens., Jan. 2010.

[5] P. Prats-Iraola et al, 'On the processing of very high- resolution spaceborne SAR data,' IEEE Trans. Geosci. Remote Sens., in review.

[6] P. Prats, et al, 'Estimation of azimuth phase undulations with multisquint processing in airborne interferometric SAR images ', IEEE Trans. Geosci. Remote Sens., Jun. 2003.

[7] B. Bräutigam, et al, 'TerraSAR-X Instrument Calibration Results and Extension for TanDEM-X', IEEE Trans. Geosci. Remote Sens., Feb. 2010.

[8] J. C. Curlander and R. N. McDonough, Synthetic Aperture Radar: Systems and Signal Processing, John Wiley \& Sons, New York, USA, 1991.

[9] M. Rodriguez-Cassola et al, 'Doppler-Related Focusing Aspects in the TOPS Imaging Mode,' Proc. IGARSS, 2013.

[10] G. Krieger, et al, 'TanDEM-X: a satellite formation for high-resolution SAR interferometry', IEEE Trans. Geosci. Remote Sens., Nov. 2007.

[11] J. Mittermayer, et al, 'Bidirectional SAR imaging mode', IEEE Trans. Geosci. Remote Sens., Jan. 2013.

[12] W. G. Carrara, Spotlight Synthetic Aperture Radar: Signal Processing Algorithms, Artech House 1995. 